\title{
Fabrication of Polymer-Supported Metal Organic Framework Membrane and Its Gas Separation Performance
}

\author{
Jingru Fu ${ }^{1}$, Teng Ben ${ }^{1}$, Shilun Qiu ${ }^{2, *}$ \\ ${ }^{1}$ College of Chemistry, Jilin University, Changchun 130012, P. R. China. \\ ${ }^{2}$ State Key Laboratory of Inorganic Synthesis and Preparation Chemistry, Jilin University, Changchun 130012, P. R. China.
}

\begin{abstract}
The fabrication of compact, continuous, and large-scale metal organic framework (MOF) membranes with high permeability and $\mathrm{H}_{2} / \mathrm{CO}_{2}$ selectivity remains challenging because of the wake interaction between the MOF membrane and the substrate. In addition, substrates with smooth and plain surfaces and suitable pore size are required to prepare high-quality MOF membranes because it is difficult to obtain dense and continuous MOF membranes on a substrate with large pores and rough surfaces. To overcome these challenges, numerous MOF membrane growth methods have emerged, including in situ (direct) growth, secondary (seeded) growth, and layer-by-layer

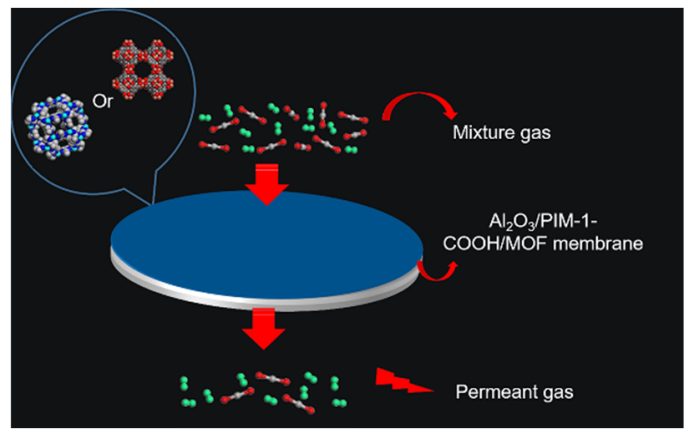
growth methods as well as electrostatic spinning and the chemical modification of the substrate. Among these methods, usage of substrates suitable for surface-functionalization is a promising technique. Herein, $\mathrm{Al}_{2} \mathrm{O}_{3}$ was selected as the substrate and was coated with PIM-1 (one polymer of intrinsic microporosity), followed by carboxylation of PIM-1 to furnish a large number of carboxyl groups on the surface. In situ growth of the MOF membrane using the interactions between the carboxyl group and the metal yielded two types of compact, continuous, and large-scale polymer-supported MOF membranes (PIM-1-COOH/ZIF-8 and PIM-1$\mathrm{COOH} / \mathrm{HKUST}$-1). Furthermore, the fabricated polymer-supported MOF membrane structures were investigated by powder X-ray diffraction (XRD) and scanning electron microscopy (SEM). Gas separation experiments were performed to explore the gas permeability and selectivity of the prepared MOF membranes. The XRD characterization confirmed the pure phase and high crystallinity of the MOF membranes. The SEM images showed that the MOF membranes were compact and continuous with a tight combination between the MOF crystal membrane and the substrate. Gas separation measurements showed that both MOF membranes exhibited high $\mathrm{H}_{2}$ permeability and selectivity for $\mathrm{H}_{2} / \mathrm{CO}_{2}$. For the PIM-1-COOH/ZIF-8 and PIM-1-COOH/HKUST-1 membranes, the $1: 1$ binary mixtures gas separation factors of $\mathrm{H}_{2} / \mathrm{CO}_{2}$ calculated as the gas molar ratios in the permeate and retentate side 7.32 and 9.69 , respectively, at room temperature and atmospheric pressure The $\mathrm{H}_{2} / \mathrm{CO}_{2}$ mixture separation factors of the two MOF membranes exceeded the corresponding Knudsen constants (4.7), with $\mathrm{H}_{2}$ permeances higher than $3.16 \times 10^{-6}$ and $1.14 \times 10^{-6} \mathrm{~mol} \cdot \mathrm{m}^{-2} \cdot \mathrm{s}^{-1} \cdot \mathrm{Pa}^{-1}$, respectively. The ideal separation factors of $\mathrm{H}_{2} / \mathrm{CO}_{2}$ of both MOF membranes calculated as the ratio of single gas permeances were 7.70 and 12.04 , respectively, with the respective $\mathrm{H}_{2}$ permeances of up to $3.73 \times 10^{-6}$ and $3.86 \times 10^{-6} \mathrm{~mol} \cdot \mathrm{m}^{-2} \cdot \mathrm{s}^{-1} \cdot \mathrm{Pa}^{-1}$. Because of their outstanding characteristics, these novel MOF membranes can be widely used in the fields of $\mathrm{H}_{2}$ purification and separation.
\end{abstract}

Key Words: Membrane; Polymer; Metal organic frameworks; Gas separation; Hydrogen purity

Received: January 29, 2019; Revised: February 25, 2019; Accepted: February 26, 2019; Published online: March 6, 2019.

${ }^{*}$ Corresponding author. Email: sqiu@jlu.edu.cn; Tel.: +86-431-85168589.

The project was supported by the National Natural Science Foundation of China $(21390394,21471065,21871103)$ and the Science and Technology

Department of Jilin Province Foundation, China (20180414009GH).

国家自然科学基金(21390394, 21471065, 21871103)和吉林省科技发展计划项目(20180414009GH)资助

(C) Editorial office of Acta Physico-Chimica Sinica 


\title{
聚合物支撑的金属有机骨架膜的制备及其气体分离性能
}

\author{
付静茹 ${ }^{1}$, 贲腾 ${ }^{1}$, 装式纶 ${ }^{2,}{ }^{*}$ \\ 1 吉林大学, 化学学院, 长春 130012 \\ 2 吉林大学, 无机合成与制备化学国家重点实验室, 长春 130012
}

\begin{abstract}
摘要：由于MOF (金属有机骨架)膜与基底之间的作用力较薄弱, 所以制备具有高的 $\mathrm{H}_{2}$ 渗透性和 $\mathrm{H}_{2} / \mathrm{CO}_{2}$ 选择性的致密连续的 大面积金属有机骨架膜仍具有巨大挑战。本文选取多孔 $\mathrm{Al}_{2} \mathrm{O}_{3}$ 作为基底, 在表面涂覆一层PIM-1 (一种固有微孔聚合物), 并对 其进行羧基化处理，使得表面具有大量的羧基基团，随后利用羧基与金属之间的相互作用，原位生长得到了两种致密连续的 聚合物支撑的MOF膜(PIM-1-COOH/ZIF-8和PIM-1-COOH/HKUST-1)。通过XRD的表征可以看出MOF膜是纯相的并且具有 较高的结晶性; SEM的测试结果表明MOF膜是致密连续的并且MOF膜与基底之间紧密结合。气体分离测试结果表明, 这两 种MOF膜对 $\mathrm{H}_{2}$ 具有较高的渗透性以及 $\mathrm{H}_{2} / \mathrm{CO}_{2}$ 选择性。在常温常压下, 对于PIM-1-COOH/ZIF-8和PIM-1-COOH/HKUST-1膜, $\mathrm{H}_{2} / \mathrm{CO}_{2}$ 双组分气体的分离系数分别为 $7.32 、 9.69$, 并且它们 $\mathrm{H}_{2}$ 的渗透通量分别高于 $3.16 \times 10^{-6} 、 1.14 \times 10^{-6} \mathrm{~mol} \cdot \mathrm{m}^{-2} \cdot \mathrm{s}^{-1} \cdot \mathrm{Pa}^{-1}$ 。 在单组份测试中, 这两种MOF膜的 $\mathrm{H}_{2} / \mathrm{CO}_{2}$ 的理想分离系数分别为 $7.70 、 12.04 ; \mathrm{H}_{2}$ 的渗透通量分别高达 $3.73 \times 10^{-6} 、 3.86 \times$ $10^{-6} \mathrm{~mol} \cdot \mathrm{m}^{-2} \cdot \mathrm{s}^{-1} \cdot \mathrm{Pa}^{-1}$ ，这就表明这两种MOF膜有望在 $\mathrm{H}_{2}$ 的纯化和分离方面广泛应用。
\end{abstract}

关键词: 膜; 聚合物; 金属有机骨架; 气体分离; 氢气纯化 中图分类号: 0641

\section{1 引言}

在过去的几十年里, 膜材料因其在气体分离 ${ }^{1-5}$, 液体分离 6,7 , 催化 ${ }^{8,9}$ 等方面具有潜在和实际的应 用价值而得以迅速的发展, 已经广泛的应用于人 们生活的各个方面。与传统的分离过程例如蒸馏 和吸附相比, 基于膜材料的分离更加具备节能和 高效性。在将来 $\mathrm{H}_{2}$ 被公认为是最有前景的能源, 这 是由于 $\mathrm{H}_{2}$ 具有优越的特性, 例如 $\mathrm{H}_{2}$ 是全宇宙中 最丰富的元素和最轻的能源, 同样它还是单位质 量最丰富的能源以及二次清洁能源 ${ }^{10,11}$ 。然而氢气 的纯化和分离严重限制了它的商业应用。而基于 膜材料分离的方法在氢气纯化领域得到了广泛的 关注, 这是由于膜材料分离的方法与其他的氢气 纯化方法相比具备更高的节能和环境友好的优 点。一直以来, 膜分离技术都被认为是高效、节能 且无污染, 而其核心就是分离膜, 分离膜的好坏直 接影响分离性能的好坏, 因此, 选择合适的材料制 备高效稳定的膜材料是十分必要的。

金属有机骨架材料(MOFs) 是由金属离子或者 金属簇通过配位键与有机配体相结合得到的。不 同的配体与不同的金属离子或者金属簇相结合，可 以得到丰富多样的拓扑结构。据报道, 在过去的 10 年, 有超过 20000 种 MOFs 被报道, 并且这个数 目还在持续增长 ${ }^{12}$ 。此外, MOFs 规整的孔尺寸, 较高的表面积以及气体吸附能力, 都使得 MOFs 成 为制备气体分离膜的理想材料。基于 MOF 的膜材
料在小分子气体分离中具备相对较强的孔尺寸篮 分效应和吸附扩散效应, 因此 MOF 膜在 $\mathrm{H}_{2}$ 的选 择性分离和纯化领域具备极大的应用前景。但是 如何制备既具有高的气体渗透通量又具备良好的 气体分离选择性的大范围的连续致密的 MOF 膜 仍是一个巨大的挑战。

目前制备 MOF 膜的方法主要有原位晶化 法 ${ }^{13-15}$, 层层(LBL)沉积法 ${ }^{16-18}$, 二次生长法 ${ }^{19-21}$, 双金属源合成法 22 等, 而这些方法很难得到大面 积的连续致密的 MOF 膜。这是由于在基底上 MOF 晶体很难异相成核, 导致 MOF 膜和基底之间的相 互作用很薄弱, 除此之外晶体与晶体之间的共生 性也比较差。人们发现要想解决这一问题, 对基底 进行化学修饰的处理 ${ }^{23,24}$ 以及晶种涂覆基底 ${ }^{25}$ 是 非常必要的, 其中尤其以基底的表面功能化修饰 效果比较好。Caro 和他的同事们 26-28 利用 3-氨基 丙基三乙氧基硅烷(APTES)修饰基底, 通过胺基共 价功能化作用制备了 ZIF-22、ZIF-90、ZIF-95 等 一系列 MOF 膜。但是 APTES 修饰基底过程复杂, 要求无水无氧苛刻的实验条件, 并且在修饰过程 中用到了甲苯这种毒性比较大的危险药品, 所以 简化基底的化学修饰方法是非常必要的。在 2012 年, 我们课题组 ${ }^{29}$ 首次利用聚合物支撑原位生长 的方法, 简单高效地制备了大面积且连续的 MOF 膜, 并且依然具有良好的气体渗透分离选择性能。 在此基础上, 本文以 $\mathrm{Al}_{2} \mathrm{O}_{3}$ 为基底, 涂覆一层固有 


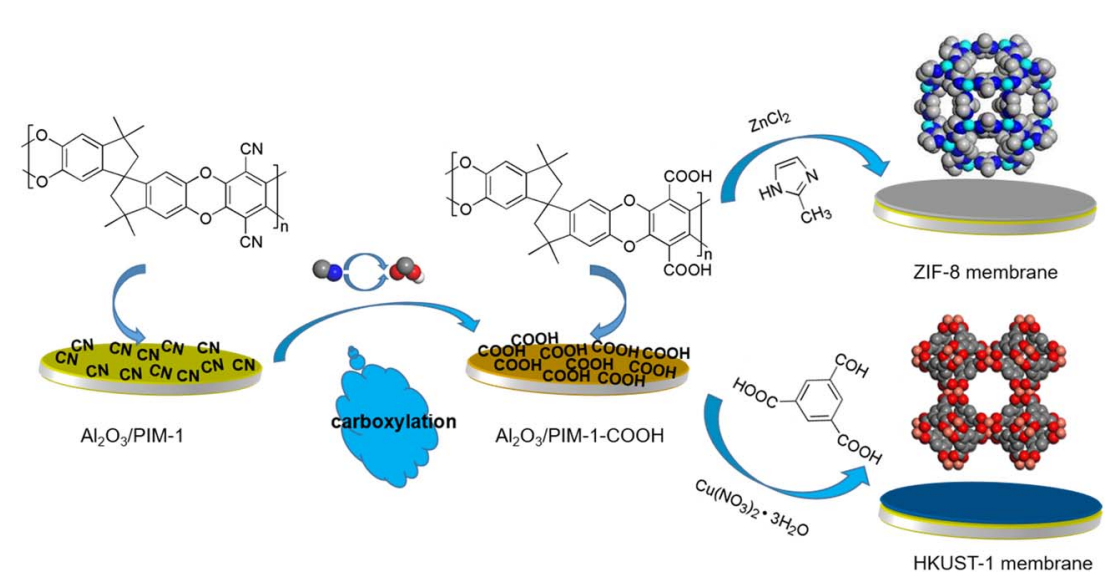

图 1 MOF 膜的制备示意图

Fig. 1 The schematic illustration of the MOF membrane.

微孔聚合物(PIM-1), 并将 PIM-1 进行羧基化处理, 利用羧基与金属之间的相互作用以达到促进 MOF 晶体在基底上异相成核的目的, 最终原位生长了 两种致密连续的 MOF 膜(PIM-1-COOH/ZIF-8 和 PIM-1-COOH/HKUST-1 膜)。具体制备流程图如图 1 所示。气体分离的测试结果表明所制备的 MOF 膜表现出了良好的气体渗透分离选择性能, 既具 有高的 $\mathrm{H}_{2}$ 渗透通量又具有较高的 $\mathrm{H}_{2} / \mathrm{CO}_{2}$ 分离 系数。

\section{2 实验部分}

\section{1 试剂与仪器}

$\mathrm{CaH}_{2}$ (99.99\%)购买于西格玛奥德利奇(中国), $5,5^{\prime}, 6,6^{\prime}$-四羟基-3,3,3',3'-四甲基-1, $1^{\prime}$-螺旋双狮满 $(>96 \%), 2,3,5,6$-四氟对苯二腈( $>98 \%$ )购买于 $\mathrm{TCI}$ 。 $\mathrm{Al}_{2} \mathrm{O}_{3}(99.99 \%), \mathrm{ZnCl}_{2}$ (ACS), $\mathrm{Cu}\left(\mathrm{NO}_{3}\right)_{2} \cdot 3 \mathrm{H}_{2} \mathrm{O}$ (99\%), 1,3,5-苯三甲酸(BTC) $(95 \%)$, 二甲基咪唑(98\%)和 甲酸钠 $(99.5 \%)$ 购于阿拉丁。 $\mathrm{NaOH}(\geq 96.0 \%)$, 甲 醇( $\mathrm{AR}, \geq 99.5 \%)$, 乙醇 $(\mathrm{AR}, \geq 99.5 \%), N, N$-二甲 基甲酰胺 $(\mathrm{AR}, \geq 99.5 \%)(\mathrm{DMF})$ 均购买于西陇化工。 氯仿 $(A R, \geq 99.0 \%$ )购买于广州化工。DMF 使用前 用 $\mathrm{CaH}_{2}$ 进行减压蒸馏。

红外测试是在日本岛津 IRAFFINITY-1 型傅 里叶变换红外光谱仪上进行, 采用 $\mathrm{KBr}$ 压片法, 扫 描范围为 $400-4000 \mathrm{~cm}^{-1}$ 。 $X$ 射线衍射是在日本 SHIMADZU XRD-6000 X-Ray 粉末 X 射线衍射仪 上进行, $\mathrm{Cu}$ 靶, 加速电压 $40 \mathrm{kV}$, 管电流 $30 \mathrm{mV}$, 扫描范围为 $4^{\circ}-40^{\circ}$, 扫描速率为 $0.3\left(^{\circ}\right) \cdot \mathrm{min}^{-1}(2 \theta)$ 。 扫描与 EDS 元素分析是在日本 JEOS JSM 6700 扫 描电子显微镜上进行, 加速电压为 $15 \mathrm{kV}$, 景深为 $10 \mathrm{~mm}$ 。

气体峰面积测试在日本 SHIMADZU GC-14C 气相色谱仪上进行, 载气为 $\mathrm{Ar}$, 检测器温度为 $120^{\circ} \mathrm{C}$, 柱箱温度为 $30^{\circ} \mathrm{C}$ 。自制气体分离装置, 与 气相色谱连用。气体分离测试, $\mathrm{He}$ 为载气, $\mathrm{H}_{2}$ 、 $\mathrm{CO}_{2} 、 \mathrm{~N}_{2} 、 \mathrm{CH}_{4}$ 为原料气, 载气流速为 $150 \mathrm{~mL} \cdot \mathrm{min}^{-1}$, 原料气流速为 $50 \mathrm{~mL} \cdot \mathrm{min}^{-1}$ 。室温下进行测试, 并 且膜两侧的压力维持在 $1 \mathrm{bar}(1 \mathrm{bar}=100 \mathrm{kPa})$ 。用 皇泡流量计测量气体流速。具体的气体分离装置 如图 2 所示。

\section{2 实验过程}

$\mathrm{Al}_{2} \mathrm{O}_{3}$ 基底的处理: 将 $\mathrm{Al}_{2} \mathrm{O}_{3}$ 基底用砂纸打磨 成直径为 $2 \mathrm{~cm}$, 厚度为 $2 \mathrm{~mm}$ 的圆片。将得到的 $\mathrm{Al}_{2} \mathrm{O}_{3}$ 片浸泡在 $50 \mathrm{~mL}$ 蒸馏水中, 超声清洗 $20 \mathrm{~min}$, 重复三次。随后用无水乙醇清洗三次, 每次 $50 \mathrm{~mL}$ 。 最后, 放到 $80^{\circ} \mathrm{C}$ 烘箱中干燥过夜。

PIM-1 的合成 ${ }^{30}$ : 将 5,5',6,6'-四羟基-3,3,3', 3'四甲基-1,1'螺旋双狮满(1.02 $\mathrm{g}, 3 \mathrm{mmol}$ ), 2,3,5,6四氟对苯二腈 $(0.60 \mathrm{~g}, 3 \mathrm{mmol})$ 以及无水 $\mathrm{K}_{2} \mathrm{CO}_{3}$ $(3.14 \mathrm{~g}, 24 \mathrm{mmol})$ 置于 $50 \mathrm{~mL}$ 两口瓶中, Ar 保护下 注入 $20 \mathrm{~mL}$ 无水 $\mathrm{DMF}$, 有黄色沉淀析出, 并在

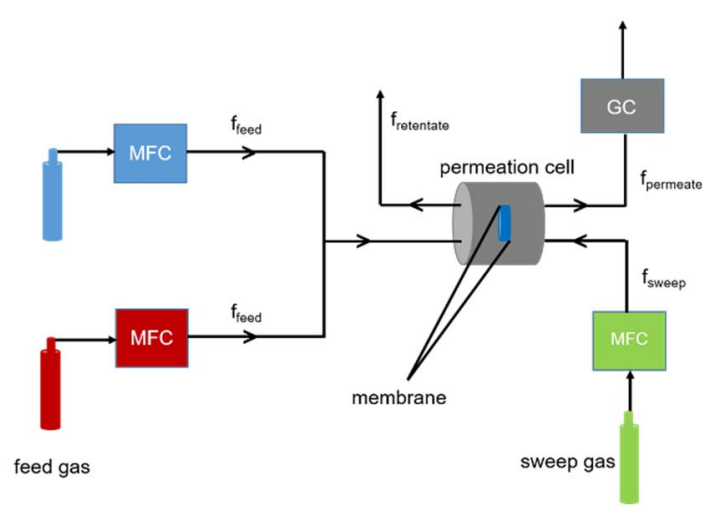

图 2 气体分离装置图

Fig. 2 Schematic illustration of gas separation set-up. MFC: Mass flow controller; GC: Gas chromatograph; f: volumetric flow rate. 
$\mathrm{Ar}$ 保护下于 $65^{\circ} \mathrm{C}$ 搅拌 $72 \mathrm{~h}$ 。反应结束后, 冷却 到室温, 倒入 $60 \mathrm{~mL}$ 水中, 搅拌 $1 \mathrm{~h}$, 过滤, 得到 粗产物。随后将得到的固体用极少量的氯仿完全 溶解, 加入大量甲醇重沉淀, 过滤, 并于 $80^{\circ} \mathrm{C}$ 抽 真空干燥 $8 \mathrm{~h}$, 得到最终产物亮黄色的 PIM-1。

$\mathrm{Al}_{2} \mathrm{O}_{3} / \mathrm{PIM}-1$ 基底和自支撑 PIM-1 膜的制备: 将 PIM-1 配成 $3 \%\left(w\right.$, 质量分数) 的 $\mathrm{CHCl}_{3}$ 溶液, 均 匀的涂覆在 $\mathrm{Al}_{2} \mathrm{O}_{3}$ 基底和玻璃片上, 为了减缓溶剂 的挥发速度, 在其上面盖上一个径口塞有棉花的 长颈玻璃漏斗, 一天以后 $\mathrm{CHCl}_{3}$ 溶剂挥发完全, 得 $\mathrm{Al}_{2} \mathrm{O}_{3} / \mathrm{PIM}-1$ 基底和自支撑的 PIM- 1 膜。

PIM-1 的羧 基化 (PIM-1-COOH) ${ }^{31}$ : 将 $\mathrm{Al}_{2} \mathrm{O}_{3} / \mathrm{PIM}-1$ 基底和自支撑的 PIM- 1 膜浸泡在 10\% $\mathrm{NaOH}$ 的乙醇/水溶液 $(V: V=9: 1)$ 中, 室温下反应 $48 \mathrm{~h}$ 。反应结束后, 用蒸馏水把基底和 PIM-1 膜清 洗至中性, 然后放入用浓 $\mathrm{HCl}$ 调 $\mathrm{PH}$ 值为 2-4 的 蒸馏水中煮沸 $1 \mathrm{~h}$, 随后再次用蒸馏水洗到中性, 最后浸泡在无水乙醇中过夜, 室温下自然干燥, 得 到羧基化的 $\mathrm{Al}_{2} \mathrm{O}_{3} / \mathrm{PIM}-1-\mathrm{COOH}$ 和 PIM-1-COOH 自支撑膜。

PIM-1-COOH 上生长 ZIF-8 膜 32 (PIM-1$\mathrm{COOH} / \mathrm{ZIF}-8)$ : 将 $\mathrm{Al}_{2} \mathrm{O}_{3} / \mathrm{PIM}-1-\mathrm{COOH}$ 浸入到 ZIF8 的母液中 $\left(\mathrm{ZnCl}_{2} ： 544.0 \mathrm{mg} ; 2\right.$-甲基咪唑：492.0 $\mathrm{mg}$; 甲酸钠: $288.0 \mathrm{mg}$; 甲醇: $41.0 \mathrm{~mL}$ ), 置于反 应釜中, $120{ }^{\circ} \mathrm{C}$ 反应 $4 \mathrm{~h}$ 。将得到的 PIM-1COOH/ZIF-8 膜用甲醇洗, 并于室温下干燥。

PIM-1-COOH 上生长 HKUST-1 膜 ${ }^{29}$ (PIM-1$\mathrm{COOH} / \mathrm{HKUST}-1)$ : 将 $\mathrm{Al}_{2} \mathrm{O}_{3} / \mathrm{PIM}-1-\mathrm{COOH}$ 浸入到 HKUST-1 的母液中(水/乙醇: $(40 \mathrm{~mL}, 1: 1, V / V)$; $\left.\mathrm{Cu}\left(\mathrm{NO}_{3}\right)_{2} \cdot 3 \mathrm{H}_{2} \mathrm{O}: 0.7 \mathrm{~g} ; \mathrm{H}_{3} \mathrm{BTC}: 0.336 \mathrm{~g}\right)$, 置于反 应反应釜中, $120^{\circ} \mathrm{C}$ 反应 $72 \mathrm{~h}$ 。将得到的 PIM-1COOH/HKUST-1 膜用乙醇洗, 并于室温下干燥。

\section{3 结果与讨论}

\section{1 自支撑 PIM-1 膜与 $\mathrm{Al}_{2} \mathrm{O}_{3} / \mathrm{PIM}-1$ 基底的羧 基化表征}

首先将 PIM-1 粉末配制成 3\%(w) 的氯仿溶 液, 涂覆在 $\mathrm{Al}_{2} \mathrm{O}_{3}$ 基底或玻璃片上, 利用溶液浇铸 的方法得到 $\mathrm{Al}_{2} \mathrm{O}_{3} / \mathrm{PIM}-1$ 基底和自支撑的 PIM-1 膜。因为 PIM-1 结构中的氰基在强碱性溶液中可 以经过水解转化为羧基, 所以我们对得到的 $\mathrm{Al}_{2} \mathrm{O}_{3} / \mathrm{PIM}-1$ 和自支撑的 PIM-1 膜进行羧基化处 理, 最终得到了 $\mathrm{Al}_{2} \mathrm{O}_{3} / \mathrm{PIM}-1-\mathrm{COOH}$ 和自支撑的 PIM-1-COOH 膜。图 3a 为 PIM-1 和 PIM-1-COOH 自支撑膜的红外图, 可以很明显的看出, PIM-1$\mathrm{COOH}$ 在 $1690 \mathrm{~cm}^{-1}$ 处以及 $3000-3600 \mathrm{~cm}^{-1}$ 处较

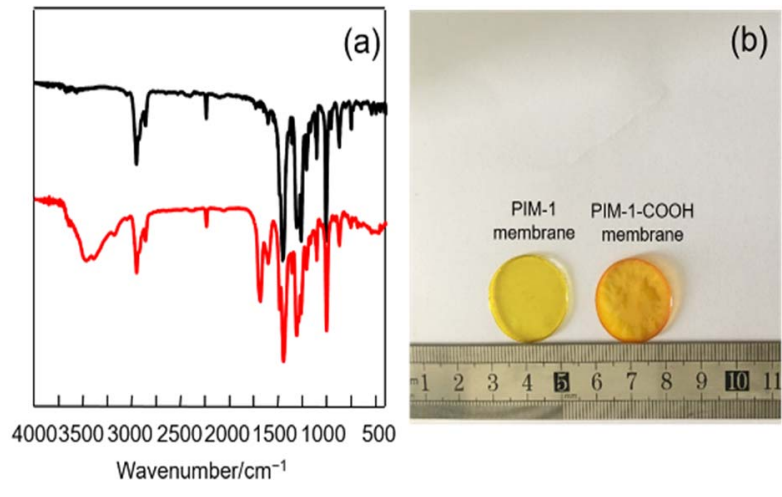

图 3 (a) PIM-1 膜(黑色)和 PIM-1-COOH 自支撑膜 (红色)的傅里叶变换红外谱图; (b) PIM-1 和

PIM-1-COOH 自支撑膜的光学照片

Fig. 3 (a) FTIR spectra of PIM-1 membrane (black) and PIM-1-COOH free-standing membrane (red);

(b) the optical pictures of PIM-1 and PIM-1-COOH free-standing membrane.

PIM-1 膜有着明显的区别。经过分析, $1690 \mathrm{~cm}^{-1}$ 可以归属于羰基伸缩振动, 3000-3600 $\mathrm{cm}^{-1}$ 则可归 属于羟基的伸缩振动, 同时 $2220 \mathrm{~cm}^{-1}$ 处氧基的特 征吸收仍然存在但是强度减弱, 表明我们成功的 在 PIM-1 膜和 $\mathrm{Al}_{2} \mathrm{O}_{3} / \mathrm{PIM}-1$ 表面部分修饰上了羧 酸基团。从图 $3 \mathrm{~b}$ 中也可以看出羧基化前后 PIM-1 膜的颜色的变化, 经过羧基化处理以后, PIM-1 颜 色加深变为黄褐色, 这表明 PIM-1 中导致表现出 亮黄色的 $\mathrm{CN}$ 基团的强共轭作用在逐渐弱化 ${ }^{31}$ 。

\section{$3.2 \mathrm{PIM}-1-\mathrm{COOH} / \mathrm{M}^{2+}\left(\mathrm{Zn}^{2+}, \mathrm{Cu}^{2+}\right)$ 的表征}

随后我们利用羧基和金属之间的相互作用, 在 $\mathrm{Al}_{2} \mathrm{O}_{3} / \mathrm{PIM}-1-\mathrm{COOH}$ 基底上原位生长了两种 $\mathrm{MOF}$ 膜, 得到了聚合物支撑的 PIM-1-COOH/ZIF-8 膜和 PIM-1-COOH/HKUST-1 膜。为了验证羧基化后的 PIM-1 和金属离子之间确实可以通过相互作用把 金属离子针定在 PIM-1 膜上。我们利用生长 MOF 的两种金属盐分别与在相同羧基化条件下得到的 PIM-1-COOH 粉末在相应的 MOF 生长条件下去 反应, 随后对所得到的 $\mathrm{PIM}-1-\mathrm{COOH} / \mathrm{Zn}^{2+}$ 以及 PIM-1-COOH $/ \mathrm{Cu}^{2+}$ 两种产物进行 EDS-Mapping 的 表征, 如图 4 所示。通过图 $4 \mathrm{a}, \mathrm{b}$, e 以及图 4c, $\mathrm{d}, \mathrm{f}$ 我们可以看出两种材料中分别含有 $\mathrm{Zn}$ 元素和 $\mathrm{Cu}$ 元素, 表明通过羧基和金属离子之间的相互作 用 ${ }^{31}$, 确实可以把金属离子引入到 PIM-1-COOH 中, 这就证实了 $\mathrm{Al}_{2} \mathrm{O}_{3} / \mathrm{PIM}-1-\mathrm{COOH}$ 基底确实可 以与 $\mathrm{MOF}$ 膜生长母液中的金属离子发生相互反 应, 随后把金属离子针定在基底上, 这一部分金属 离子接着再与配体发生配位作用, 从而参与到 $\mathrm{MOF}$ 晶体的生长过程中。具体的反应过程如示意 

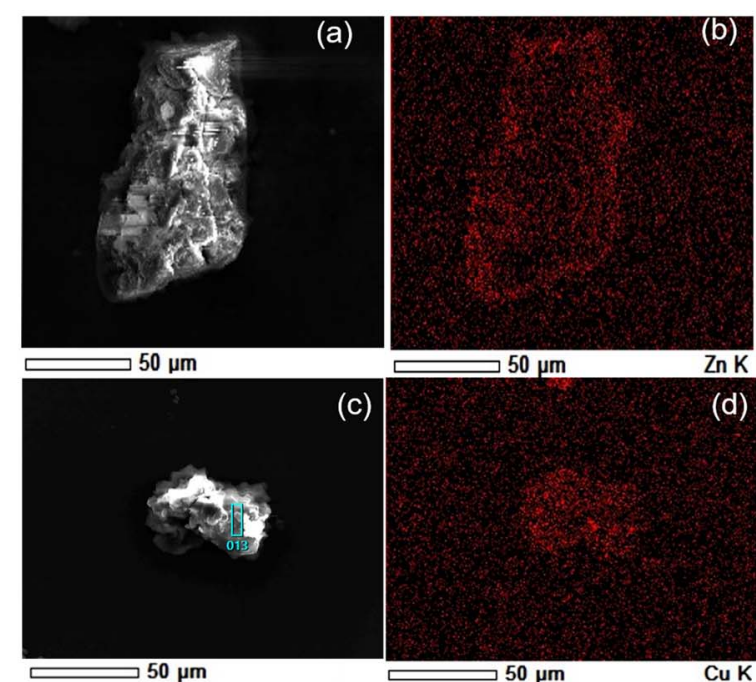

)
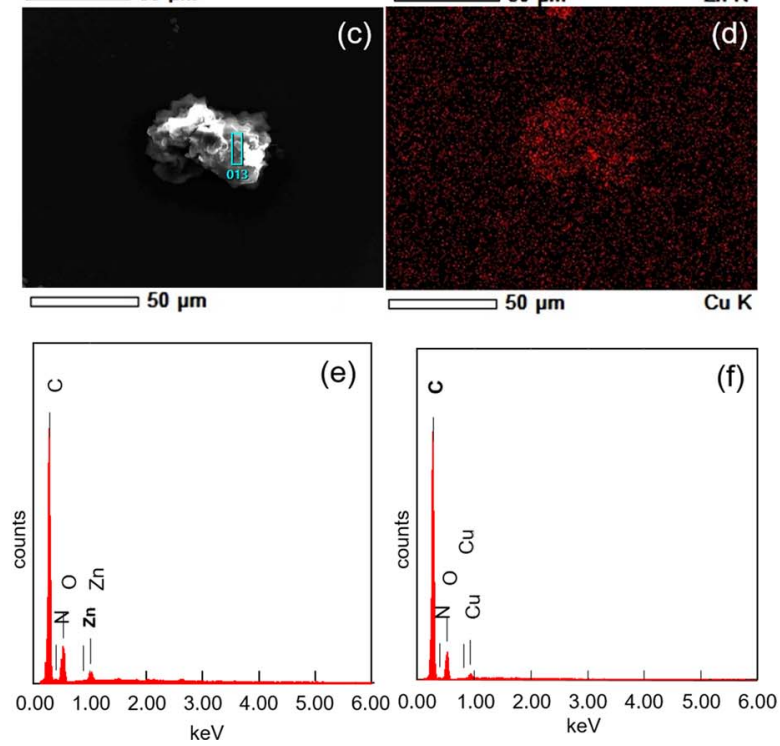

图 4 PIM-1-COOH $/ \mathrm{Zn}^{2+}(\mathrm{a}, \mathrm{b}, \mathrm{e})$ 和 $\mathrm{PIM}-1-\mathrm{COOH} / \mathrm{Cu}^{2+}(\mathrm{c}, \mathrm{d}, \mathrm{f})$ 的元素分析

Fig. 4 The EDS-Mapping spectrum of the PIM-1$\mathrm{COOH} / \mathrm{Zn}^{2+}(\mathrm{a}, \mathrm{b}, \mathrm{e})$ and PIM-1-COOH$/ \mathrm{Cu}^{2+}(\mathrm{c}, \mathrm{d}, \mathrm{f})$.

图 1 所示。

\section{$3.3 \mathrm{PIM}-1-\mathrm{COOH} / \mathrm{MOF}$ 膜的表征}

图 5a 是 $\mathrm{Al}_{2} \mathrm{O}_{3} / \mathrm{PIM}-1$ 和 $\mathrm{Al}_{2} \mathrm{O}_{3} / \mathrm{PIM}-1-\mathrm{COOH}$ 基底的光学照片, 从图中我们可以清楚地看到涂 覆在 $\mathrm{Al}_{2} \mathrm{O}_{3}$ 基底上的 PIM-1 颜色在羧基化前后发 生了明显的变化, 说明即使把 PIM-1 涂覆在基底 上同样可以同自支撑的 PIM-1 膜一样发生着基化 反应。从图 $5 \mathrm{~b}$ 可以看出, 白色和蓝色的 MOF 层 把基底上的 PIM-1-COOH 层完全覆盖, 同时也没 有肉眼可见的裂纹缺陷, 说明我们制备的 MOF 膜 整体上是致密连续的。图 $5 \mathrm{c}$ 是 $\mathrm{Al}_{2} \mathrm{O}_{3} / \mathrm{PIM}-1-\mathrm{COOH}$ 基底的 SEM 照片, 从中我们可以看到其表面平整 光滑, 并且除了少有的裂缝绝大部分看不出大孔 的存在, 说明通过涂覆小孔的聚合物可以去调控 大孔的基底并且使其表面平整光滑, 而稍有的裂 缝也保证了基底不会阻碍气体透过 MOF 膜而牺 牲气体渗透性。为了表征 MOF 膜的结晶性, 我们 对实验所得到的两种 MOF 膜以及 MOF 粉末分别 进行了 XRD 的测试。图 $5 \mathrm{f}$ 为合成的 PIM-1COOH/ZIF-8 膜与 ZIF-8 粉末的 XRD 衍射图, 结

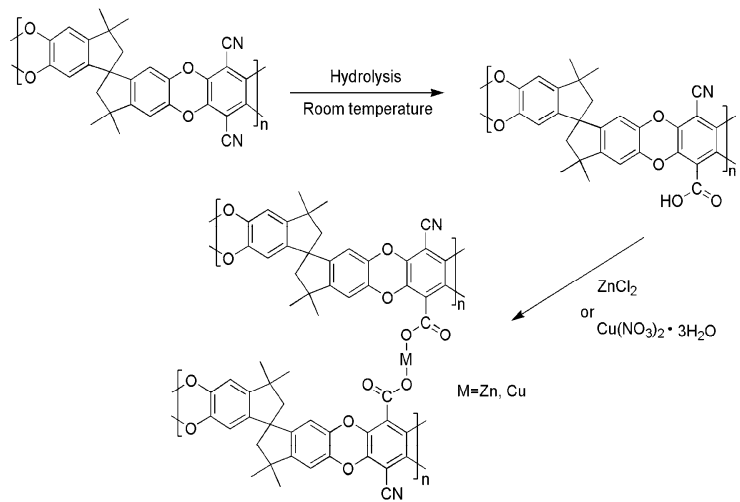

示意图 1 PIM-1-COOH $/ \mathrm{M}^{2+}$ 的合成

Scheme 1 Synthesis of PIM-1-COOH/ $/ \mathrm{M}^{2+}$.

果表明合成的 ZIF-8 膜的 XRD 与 ZIF-8 粉末的 $X R D$ 可以很好地相吻合, 且没有多余的杂峰, 证 明我们成功的制备出了 PIM-1-COOH/ZIF-8 膜, 并 且所得到的 PIM-1-COOH/ZIF-8 膜是由 ZIF-8 晶 体组成的纯相。同样地, PIM-1-COOH/HKUST-1 膜 的 XRD 与 HKUST-1 粉末 XRD 相一致(如图 5i), 表明我们也得到了纯相的且具有高的结晶性的 PIM-1-COOH/HKUST-1 膜。

为了进一步表征 MOF 膜的形貌以及观察 MOF 晶体之间的共生性和 MOF 膜和基底之间的 连接紧密性, 我们对 MOF 膜的正面与截面均进行 了 SEM 的测试。如图 5d 和 $5 \mathrm{~g}$ 所示, 从图上我们 可以很明显的看到 ZIF-8 和 HKUST-1 的晶体并不 是随机离散地分布在基底上而是排列紧密, 没有 明显的针孔或裂纹, 表明这两种 MOF 膜生长致密 连续且共生性良好。这是由于基底上聚合物层含 有丰富的羧基, 在 MOF 膜生长的初始阶段, 羧基 首先与 $\mathrm{MOF}$ 膜生长母液中的金属离子之间发生 相互作用, 利用配位把大量的金属离子牢牢地针 定在基底上, 这一部分金属离子再继续与配体发 生配位作用而参与到 $\mathrm{MOF}$ 晶体的成核与生长过 程中, 最终形成致密连续的 MOF 膜。图 $5 \mathrm{e}, \mathrm{h}$ 分 别是 PIM-1-COOH/ZIF-8 和 PIM-1-COOH/HKUST1 膜的截面图, 膜的厚度分别为 70 和 $72 \mu \mathrm{m}$ 。从 中我们还可以看到 MOF 膜与基底之间连接紧密, 没有出现裂缝或断痕, 说明 MOF 膜和基底之间具 有强的结合力, 这是由于基底上的大量的羧基为 MOF 晶体在基底上的生长提供了异相成核的位 点, 羧基与金属离子之间具有强的相互作用力。这 就保障了所制备的 MOF 膜具有良好的气体分离 选择性能。

\section{$3.4 \mathrm{PIM}-1-\mathrm{COOH} / \mathrm{MOF}$ 膜分离性能}

为了研究实验所得的 MOF 膜的分离性能, 我 们分别测试了 PIM-1-COOH/ZIF-8 膜和 PIM-1- 

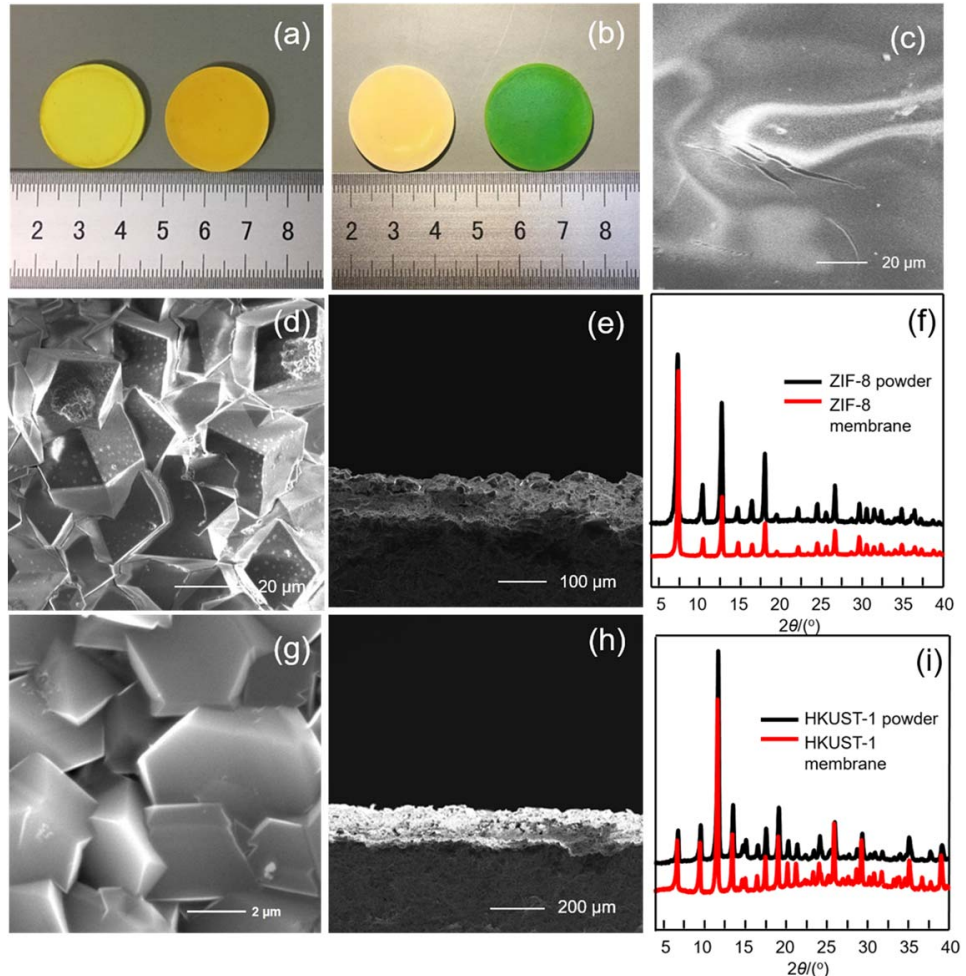

图 5 (a) $\mathrm{Al}_{2} \mathrm{O}_{3} / \mathrm{PIM}-1$ (左)和 $\mathrm{Al}_{2} \mathrm{O}_{3} / \mathrm{PIM}-1-\mathrm{COOH}$ (右)的光学照片; (b) PIM-1-COOH/ZIF-8 膜(左)和 PIM-1-COOH/HKUST-1 膜的光学照片(右); (c) $\mathrm{Al}_{2} \mathrm{O}_{3} / \mathrm{PIM}-1$ 基底的扫描图; (d, e, f) PIM-1-COOH/ZIF-8 膜的 正面和截面扫描图以及 XRD 图; (g, h, i) PIM-1-COOH/HKUST-1 膜的正面和截面扫描图以及 XRD 图

Fig. 5 (a) Optical images of $\mathrm{Al}_{2} \mathrm{O}_{3} / \mathrm{PIM}-1$ (left) and $\mathrm{Al}_{2} \mathrm{O}_{3} / \mathrm{PIM}-1-\mathrm{COOH}$ (right); (b) Optical images of

PIM-1-COOH/ZIF-8 membrane (left) and PIM-1-COOH/HKUST-1 membrane (right); (c) SEM picture of $\mathrm{Al}_{2} \mathrm{O}_{3} / \mathrm{PIM}-1$ support; (d, e, f) SEM top view, cross-section view and XRD patterns for PIM-1-COOH/ZIF-8 membrane; (g, h, i) SEM top view, cross-section view and XRD patterns for PIM-1-COOH/HKUST-1 membrane.
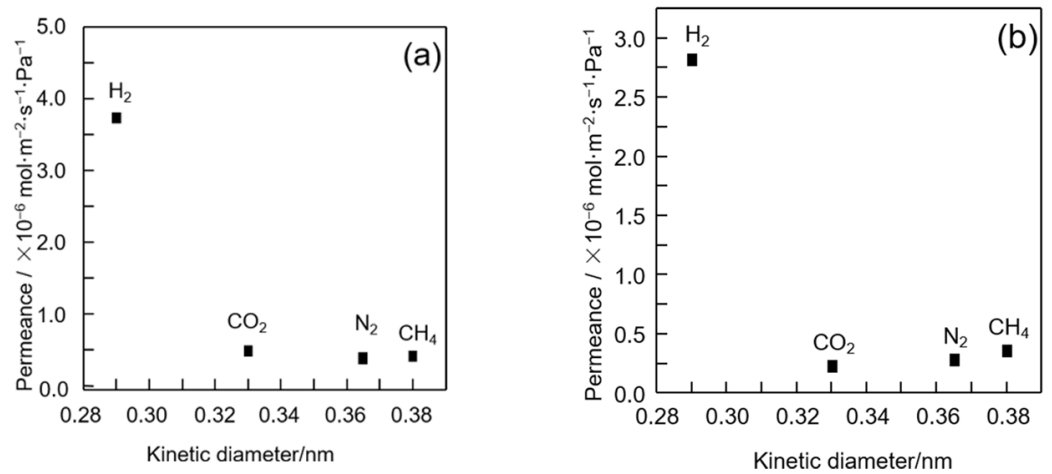

图 6 常温常压下, 单组份气体透过 PIM-1-COOH/ZIF-8 膜(a)和 PIM-1-COOH/HKUST-1 膜(b)的 渗透通量与相应的动力学直径之间的关系

Fig. 6 Single gas permeance of various gases through the (a) PIM-1-COOH/ZIF-8 membrane and (b) PIM-1$\mathrm{COOH/HKUST-1} \mathrm{membrane} \mathrm{at} \mathrm{room} \mathrm{temperature} \mathrm{and} \mathrm{atmospheric} \mathrm{pressure} \mathrm{as} \mathrm{a} \mathrm{function} \mathrm{of} \mathrm{their} \mathrm{kinetic} \mathrm{diameter.}$

$\mathrm{COOH} / \mathrm{HKUST}-1$ 膜对 $\mathrm{H}_{2} 、 \mathrm{CO}_{2} 、 \mathrm{~N}_{2}$ 和 $\mathrm{CH}_{4}$ 四种单 组分气体的渗透性能以及体积比 $1: 1 \mathrm{H}_{2} / \mathrm{CO}_{2}$ 、 $\mathrm{H}_{2} / \mathrm{N}_{2} 、 \mathrm{H}_{2} / \mathrm{CH}_{4}$ 混合气体的渗透分离选择性。图 $6 \mathrm{a}$, $\mathrm{b}$ 分 别 为 PIM-1-COOH/ZIF-8 膜 和 PIM-1$\mathrm{COOH} / \mathrm{HKUST}-1$ 膜对不同单组份气体的渗透性与 其动力学直径的关系。 $\mathrm{H}_{2}, \mathrm{CO}_{2}, \mathrm{~N}_{2}$ 和 $\mathrm{CH}_{4}$ 四种
气体的动力学直径分别为 $0.29,0.33,0.36,0.38$ $\mathrm{nm}$ 。由图 6a 可知, 常温常压下, 四种气体透过 PIM1-COOH/ZIF-8 膜的通量大小顺序为 $\mathrm{H}_{2}>\mathrm{CO}_{2}>$ $\mathrm{CH}_{4}>\mathrm{N}_{2}$ 。 $\mathrm{H}_{2}$ 的通量为 $3.73 \times 10^{-6} \mathrm{~mol} \cdot \mathrm{m}^{-2} \cdot \mathrm{s}^{-1} \cdot \mathrm{Pa}^{-1}$, $\mathrm{CO}_{2}, \mathrm{~N}_{2}$ 和 $\mathrm{CH}_{4}$ 的通量分别为 $4.85 \times 10^{-7}, 3.83 \times$ $10^{-7}, 4.17 \times 10^{-7} \mathrm{~mol} \cdot \mathrm{m}^{-2} \cdot \mathrm{s}^{-1} \cdot \mathrm{Pa}^{-1}$ 。 $\mathrm{H}_{2}$ 的通量明显 
大于 $\mathrm{CO}_{2} 、 \mathrm{~N}_{2}$ 和 $\mathrm{CH}_{4}$, 这可以归因于 $\mathrm{H}_{2}$ 的动力学 直径较小, 较其他气体更容易通过膜材料。其他气 体的渗透基本符合尺寸篮分效应, $\mathrm{CH}_{4}$ 与 $\mathrm{N}_{2}$ 的动 力学直径较大, 所以渗透通量要低于动力学直径 较小的 $\mathrm{CO}_{2}$, 也从侧面说明了 PIM-1-COOH/ZIF8 膜的致密性良好, 没有明显的缺陷, 尺寸篮分效 应在气体渗透分离中占主导作用。膜材料的理想 分离系数由两种气体的渗透通量比而求得, 而混 合双组分气体的分离系数由两种气体在渗透端和 残留端的摩尔分数之比求得。通过计算, PIM-1$\mathrm{COOH} / \mathrm{ZIF}-8$ 膜的 $\mathrm{H}_{2} / \mathrm{CO}_{2}$ 理想分离系数为 $7.70,1$ : $1 \mathrm{H}_{2} / \mathrm{CO}_{2}$ 混合气体的分离系数为 7.32 (表 1), 均高 于努森扩散系数 $\left(\mathrm{H}_{2} / \mathrm{CO}_{2}: 4.7\right)$, 并且超过了目前很 多由 ZIF-8 合成的膜, 例如, Jeong 等人 33 在 $\alpha$-氧 化铝基底上生长的 ZIF-8 膜 $\left(\mathrm{H}_{2} / \mathrm{CO}_{2}\right.$ : 3.89), Lai 等人 34 通过晶种生长的方法在氧化铝基底上制备 的 ZIF-8 膜 $\left(\mathrm{H}_{2} / \mathrm{CO}_{2}: 2.57\right)$ 以及 Banerjee 等人 ${ }^{35}$ 在 多孔聚砜基底上制备的 ZIF-8@PSF 复合膜 $\left(\mathrm{H}_{2} / \mathrm{CO}_{2}\right.$ : 3.8)等等。同时, $\mathrm{H}_{2} / \mathrm{N}_{2}$ 和 $\mathrm{H}_{2} / \mathrm{CH}_{4}$ 的理想 分离系数分别为 9.7 和 8.9 , 也高于相应的努森扩 散系数 $\left(\mathrm{H}_{2} / \mathrm{N}_{2}: 3.7 ; \mathrm{H}_{2} / \mathrm{CH}: 2.8\right)$, 并且超过了我们 组之前所报道 ${ }^{29}$ 的 PMMA-PMAA/HKUST-1 膜 $\left(\mathrm{H}_{2} / \mathrm{N}_{2}\right.$ : 5.08; $\mathrm{H}_{2} / \mathrm{CH}_{4}$ : 7.93), Qiu 等人 22 利用双 铜源制备的 $\mathrm{Cu}_{3}(\mathrm{BTC})_{2}$ 膜 $\left(\mathrm{H}_{2} / \mathrm{N}_{2}: 4.60 ; \mathrm{H}_{2} / \mathrm{CH}_{4}\right.$ : 7.8)等, 由此可知, 所制备的 PIM-1-COOH/ZIF-8 膜具有优异的气体渗透分离性能, 通过尺寸篮分 可以实现 $\mathrm{H}_{2}$ 对 $\mathrm{CO}_{2} 、 \mathrm{~N}_{2}$ 和 $\mathrm{CH}_{4}$ 的分离, 达到氢气 纯化和分离的目的。

随后我们对合成的另外一种 PIM-1COOH/HKUST-1 膜进行了分离性能的研究, PIM1-COOH/HKUST-1 膜对不同气体的渗透性与其动 力学直径的关系如图 $6 \mathrm{~b}$ 所示, 常温常压下, 四种 气体透过 PIM-1-COOH/HKUST-1 膜的通量大小 顺序为 $\mathrm{H}_{2}>\mathrm{CH}_{4}>\mathrm{N}_{2}>\mathrm{CO}_{2}$ 。 $\mathrm{H}_{2}, \mathrm{CH}_{4}, \mathrm{~N}_{2}$ 和 $\mathrm{CO}_{2}$ 的通量依次为 $3.86 \times 10^{-6}, 7.42 \times 10^{-7}, 5.82 \times 10^{-7}$,

\section{表 1 常温常压下, PIM-1-COOH/ZIF-8 的 $1: 1$ 混合气体的分离性能}

Table 1 The mixture gas $(1: 1)$ separation properties of PIM-1-COOH/ZIF-8 membrane at room temperature and atmospheric pressure.

\begin{tabular}{ccccc}
\hline Gas $(\mathrm{i} / \mathrm{j})$ & KC & Permeance $_{(\mathrm{i})}{ }^{\text {a }}$ & Permeance $_{(\mathrm{j})}{ }^{\text {a }}$ & SF \\
\hline $\mathrm{H}_{2} / \mathrm{CO}_{2}$ & 4.7 & 39.6 & 5.41 & 7.32 \\
$\mathrm{H}_{2} / \mathrm{N}_{2}$ & 3.7 & 38.4 & 8.19 & 5.63 \\
$\mathrm{H}_{2} / \mathrm{CH}_{4}$ & 2.8 & 37.6 & 1.15 & 3.92 \\
\hline
\end{tabular}

KC: Knudsen constant; SF: Selectivity factor;

a Permeance: $\times 10^{-7} \mathrm{~mol} \cdot \mathrm{m}^{-2} \cdot \mathrm{s}^{-1} \cdot \mathrm{Pa}^{-1}$.
$3.87 \times 10^{-7} \mathrm{~mol} \cdot \mathrm{m}^{-2} \cdot \mathrm{s}^{-1} \cdot \mathrm{Pa}^{-1}$ 。 $\mathrm{H}_{2}$ 有着最高的通量, $\mathrm{CO}_{2}$ 有着最低的通量, 这是因为 $\mathrm{H}_{2}$ 的动力学直径 较小, 另一方面在常温下 HKUST-1 对 $\mathrm{H}_{2}$ 的吸附 作用较弱, 所以对于 $\mathrm{H}_{2}$ 而言, 尺寸篮分效应占主 导地位, 导致 $\mathrm{H}_{2}$ 比较容易透过; 而 $\mathrm{CO}_{2}$ 分子中含 有孤对电子, 能够增强与 HKUST-1 中的路易斯酸 位点的相互作用, 也就是说该 MOF 对 $\mathrm{CO}_{2}$ 具有选 择性吸附作用, 在 $\mathrm{CO}_{2}$ 气体渗透过 $\mathrm{MOF}$ 膜的过程 中会被吸附在 MOF 材料的孔道中, 这样就阻碍了 气体 $\mathrm{CO}_{2}$ 的顺利渗透, 因此 $\mathrm{CO}_{2}$ 动力学直径比 $\mathrm{N}_{2}$ 和 $\mathrm{CH}_{4}$ 小, 却比较难透过。这也就是接下来 $\mathrm{H}_{2} / \mathrm{CO}_{2}$ 的分离系数要高于 $\mathrm{H}_{2} / \mathrm{N}_{2}, \mathrm{H}_{2} / \mathrm{CH}_{4}$ 的原因。

经过计算, $\mathrm{H}_{2} / \mathrm{CO}_{2} 、 \mathrm{H}_{2} / \mathrm{N}_{2}$ 和 $\mathrm{H}_{2} / \mathrm{CH}_{4}$ 的理想 分离系数分别为 $12.04 、 9.69$ 和 7.77 , 相对应的 1 : 1 混合气体分离系数依次为 9.69、7.03 和 5.27 (表 $2)$, 均远远高于努森扩散系数 $\left(\mathrm{H}_{2} / \mathrm{CO}_{2}: 4.7 ; \mathrm{H}_{2} / \mathrm{N}_{2}\right.$ : 3.7; $\mathrm{H}_{2} / \mathrm{CH}_{4}$ : 2.8), 也超过了许多之前报道过的 HKUST-1 膜, 例如 $\mathrm{H}_{2} / \mathrm{CO}_{2}$ 和 $\mathrm{H}_{2} / \mathrm{N}_{2}$ 的理想分离系 数超过了我们组之前发表 ${ }^{29}$ 的 PMMA-PMAA/ HKUST-1 $\left(\mathrm{H}_{2} / \mathrm{CO}_{2}: 8.68 ; \mathrm{H}_{2} / \mathrm{N}_{2}: 5.08\right), \mathrm{H}_{2} / \mathrm{CO}_{2}$ 、 $\mathrm{H}_{2} / \mathrm{N}_{2}$ 和 $\mathrm{H}_{2} / \mathrm{CH}_{4}$ 均超过了 $\mathrm{Xu}$ 等人 ${ }^{21}$ 利用晶种法 在氧化铝基底上二次生长的 HKUST- 1 膜 $\left(\mathrm{H}_{2} / \mathrm{CO}_{2}\right.$ : 5.1; $\mathrm{H}_{2} / \mathrm{N}_{2}: 3.7 ; \mathrm{H}_{2} / \mathrm{CH}_{4}: 2.9$ ), Jeong 等人 36 利 用晶种法在氧化铝基底上二次生长的 HKUST- 1 膜 $\left(\mathrm{H}_{2} / \mathrm{CO}_{2}\right.$ : 3.5; $\left.\mathrm{H}_{2} / \mathrm{N}_{2}: 3.7 ; \mathrm{H}_{2} / \mathrm{CH}_{4}: 2.4\right)$ 等, 表明 致密连续的 PIM-1-COOH/HKUST-1 膜同样具有 很好的气体分离选择性能, 可以用于 $\mathrm{H}_{2}$ 的分离和 纯化。

值得说明的是, 通过对这两种 MOF 膜进行气 体渗透分离的测试我们可以看出, 它们气体的渗 透通量要比文献 37,38 报道的在高压下测试的自支 撑的 PIM-1 膜要大很多, 这是由于最初在氧化铝 基底上涂布的那一层 PIM-1 膜是不连续的, 只是 通过羧基化处理后在整个 MOF 膜的生长过程中 起到与金属离子相互配位链接的作用, 而基底上 的羧基的量又是丰富的, 可以为 MOF 膜在基底上

表 2 常温常压下, PIM-1-COOH/HKUST-1 的 $1: 1$ 混合气体的分离性能

Table 2 The mixture gas $(1: 1)$ separation properties of PIM-1-COOH/HKUST-1 membrane at room temperature and atmospheric pressure.

\begin{tabular}{ccccc}
\hline Gas $(\mathrm{i} / \mathrm{j})$ & $\mathrm{KC}$ & Permeance $_{(\mathrm{i})}{ }^{\mathrm{a}}$ & Permeance $_{(\mathrm{j})}{ }^{\mathrm{a}}$ & $\mathrm{SF}$ \\
\hline $\mathrm{H}_{2} / \mathrm{CO}_{2}$ & 4.7 & 11.4 & 1.97 & 9.69 \\
$\mathrm{H}_{2} / \mathrm{N}_{2}$ & 3.7 & 11.5 & 1.89 & 7.03 \\
$\mathrm{H}_{2} / \mathrm{CH}_{4}$ & 2.8 & 11.9 & 2.71 & 5.27 \\
\hline
\end{tabular}

a Permeance: $\times 10^{-7} \mathrm{~mol} \cdot \mathrm{m}^{-2} \cdot \mathrm{s}^{-1} \cdot \mathrm{Pa}^{-1}$. 
的生长提供足够多的异相成核的位点, 所以这一 部分小裂痕不会影响整个 MOF 膜的连续性, 也不 会阻碍气体的渗透。通过 PIM-1 在基底上浇铸成 有缺陷的膜, 而后经过羧基化处理为 $\mathrm{MOF}$ 膜生长 这就既保证了 $\mathrm{MOF}$ 膜的致密连续性也避免了渗 透通量的降低, 进而增强了 $\mathrm{MOF}$ 膜的渗透选择 性。从图 $5 \mathrm{c}$ 中我们也可以看出 $\mathrm{Al}_{2} \mathrm{O}_{3} / \mathrm{PIM}-1$ 基底 上的平整的 PIM-1 层有少量的微米级裂痕存在。作 为对比我们在玻璃片上同样利用浇铸 PIM-1 的氯 仿溶液的方法得到了自支撑的连续的 PIM-1 膜(如 图 $1 \mathrm{~b}$ 所示), 这是由于玻璃片较 $\mathrm{Al}_{2} \mathrm{O}_{3}$ 基底平整并 且没有孔, 表面张力较小, 更有利于 PIM-1 成膜。

\section{4 结论}

总之, 我们利用 $\mathrm{Al}_{2} \mathrm{O}_{3}$ 作为基底, 通过溶液浇 铸的方法在表面涂覆形成一层不连续的平整的 PIM-1 膜, 并对其进行羧基化处理, 使得表面具有 大量的羧基基团，随后利用羧基与金属之间的相 互作用, 为 $\mathrm{MOF}$ 膜在基底上的生长提供了异相成 核的位点, 最终原位生长得到了两种致密连续的 $\mathrm{PIM}-1-\mathrm{COOH} / \mathrm{MOF}$ 膜。经过气体分离测试结果表明, PIM-1-COOH/ZIF-8 膜和 PIM-1-COOH/HKUST-1 膜对 $\mathrm{H}_{2} / \mathrm{CO}_{2}$ 混合气体的分离系数分别为 7.32 和 9.69 , 理想分离系数分别为 7.70 和 12.04 , 均远远 超过了 $\mathrm{H}_{2} / \mathrm{CO}_{2}$ 的努森系数(4.7), 并且具有比较高 的 $\mathrm{H}_{2}$ 的渗透通量, 依次为 $3.73 \times 10^{-6}$ 及 $3.86 \times$ $10^{-6} \mathrm{~mol} \cdot \mathrm{m}^{-2} \cdot \mathrm{s}^{-1} \cdot \mathrm{Pa}^{-1}$ 。表明两种 $\mathrm{MOF}$ 膜对 $\mathrm{H}_{2}$ 具 有较好的渗透分离选择性, 使其在 $\mathrm{H}_{2}$ 的分离和纯 化方面具有一定潜在的应用价值。

\section{References}

(1) Venna, S. R.; Carreon, M. A. J. Am. Chem. Soc. 2010, 132, 76. doi: $10.1021 / \mathrm{ja} 909263 \mathrm{x}$

(2) Huang, A. S.; Chen, Y.; Liu, Q.; Wang, N.; Jiang, J.; Caro J. J. Membr. Sci. 2014, 454, 126. doi: 10.1016/j.memsci.2013.12.018

(3) Zhao, Z. X.; Ma, X. L.; Kasik, A.; Li, Z.; Lin, Y. S. Ind. Eng. Chem. Res. 2013, 52, 1102. Doi: 10.1021/ie202777q

(4) Bux, H.; Feldhoff, A.; Cravillon, J.; Wiebcke, M.; Li Y. S.; Caro J. Chem. Mater. 2011, 23, 2262. doi: 10.1021/cm200555s

(5) Nian, P.; Li, Y. J.; Zhang, X.; Cao, Y.; Liu, H. O.; Zhang, X. F. ACS Appl. Mater. Interfaces 2018, 10, 4151. doi: $10.1021 /$ acsami.7b17568

(6) Wang, W. J.; Dong, X. L.; Nan, J. P.; Jin, W. Q.; Hu, Z. Q.; Chen, Y. F.; Jiang, J. W. Chem. Commun. 2012, 48, 7022. doi: $10.1039 / \mathrm{c} 2 \mathrm{cc} 32595 \mathrm{k}$

(7) Dong, X. L.; Lin, Y. S. Chem. Commun. 2013, 49, 1196. doi: $10.1039 / \mathrm{C} 2 \mathrm{CC} 38512 \mathrm{~K}$

(8) Wang, C.; deKrafft, K. E.; Lin, W. B. J. Am. Chem. Soc. 2012, 134, 7211. doi: 10.1021/ja300539p

(9) Maina, J. W.; Schutz, J. A.; Grundy, L.; Ligneris, E. D.; Yi, Z. F.; Kong, L. X.; Pozo-Gonzalo, C.; Ionescu, M.; Dumee, L. F. ACS Appl. Mater. Interfaces 2017, 9, 35010. doi: 10.1021/acsami.7b11150

(10) Jain, I. P. J. Hydrog. Energy 2009, 34, 7368. doi: 10.1016/j.ijhydene.2009.05.093

(11) Berry, G. D.; Pasternak, A. D.; Bambach, G. D.; Ray, Smith, J.; Schock, R. N. Energy 1996, 21, 289. doi: 10.1016/0360-5442(95)00104-2

(12) Furukawa, H.; Cordova, K. E.; O'Keeffe, M.; Yaghi, O. M. Science 2013, 341, 1230444. doi: 10.1126/science. 1230444

(13) Liu, Y. Y.; Ng, Z. F.; Khan, K. A.; Jeong, H. K.; Ching, C. B.; Lai, Z. P. Microporous Mesoporous Mater. 2009, 118, 296. doi: 10.1016/j.micromeso.2008.08.054

(14) Liu, Y. Y.; Hu, E. P.; Khan, E. A.; Lai, Z. P. J. Membr. Sci. 2010, 353, 36. doi: 10.1016/j.memsci.2010.02.023

(15) Zhuang, J. L.; Terfort, A.; Wöll, C. Coord. Coordination Chem. Rev. 2016, 307, 391. doi: 10.1016/j.ccr.2015.09.013

(16) Makiura, R.; Motoyama, S.; Umemura, Y.; Yamanake, H.; Sakata, O.; Kitagawa, H. Nat. Mater. 2010, 9, 565. doi: 10.1038/nmat2769

(17) Shekhah, O.; Wang, H.; Kowarik, S.; Schreiber, F.; Paulus, M.; Tolan, M.; Sternemann, C.; Evers, F.; Zacher, D.; Fischer, R. A.; et al. J. Am. Chem. Soc. 2007, 129, 15118. doi: 10.1021/ja076210u

(18) Nijem, N.; Fürsich, K.; Kelly, S. T.; Swain, C.; Leone, S. R.; Gilles, M. K. Cryst. Growth Des. 2015, 15, 2948. doi: 10.1021/acs.cgd.5b00384

(19) Ranjan, R.; Tsapatsis, M. Chem. Mater. 2009, 21, 4920. doi: $10.1021 / \mathrm{cm} 902032 \mathrm{y}$

(20) Hu, Y. X.; Dong, X. L.; Nan, J. P.; Jin, W. Q.; Ren, X. M.; Xu, N. P.; Lee, Y. M. Chem. Commun. 2011, 47, 737. doi: 10.1039/c0cc03927f

(21) Nan, J. P.; Dong, X. L.; Wang, W. J.; Jin, W. Q.; Xu, N. P. Langmuir 2011, 27, 4309. doi: 10.1021/1a200103w

(22) Guo, H. L.; Zhu, G. S.; Hewitt, I. J.; Qiu, S. L. J. Am. Chem. Soc. 2009, 131, 1646. doi: 10.1021/ja8074874

(23) Zacher, D.; Baunemann, A.; Hermes, S.; Fischer, R. A. J. Mater. Chem. 2007, 17, 2785. doi: 10.1039/B703098C

(24) Biemmi, E.; Scherb, C.; Bein, T. J. Am. Chem. Soc. 2007, 129, 8054. doi: $10.1021 / \mathrm{ja} 0701208$

(25) Li, Y. S.; Liang, F. Y.; Bux, H.; Feldhoff, A.; Yang, W. S.; Caro, J. Angew. Chem. Int. Ed. 2010, 49, 548. doi: 10.1002/anie.200905645

(26) Huang, A. S.; Bux, H.; Steinbach, F.; Caro, J. Angew. Chem. Int. Ed. 2010, 49, 4958. doi: 10.1002/anie.201001919

(27) Huang, A. S.; Wang, N.; Kong, C. L.; Caro, J. Angew. Chem. Int. Ed. 2012, 51, 10551. doi: 10.1002/anie.201204621 
(28) Huang, A. S.; Chen, Y. F.; Wang, N.; Hu, Z. Q.; Jiang, J.; Caro, J. Chem. Commun. 2012, 48, 10981. doi: 10.1039/C2CC35691K

(29) Ben, T.; Lu, C. J.; Pei, C. Y.; Xu, S. X.; Qiu, S. L. Chem. Eur. J. 2012, 18, 10250. doi: 10.1002/chem.201201574

(30) Budd, P. M.; Elabas, E. S.; Ghanem, B. S.; Makhseed, S.; Mckeown, N. B.; Msayib, K. J.; Tattershall, C. E.; Wang, D. Adv. Mater. 2004, 16, 456. doi: 10.1002/adma.200306053

(31) Zhao, H. Y.; Xie, Q.; Ding, X. L.; Chen, J. M.; Hua, M. M.; Tan, X. Y.; Zhang, Y. Z. J. Membr. Sci. 2016, 514, 305. doi: 10.1016/j.memsci.2016.05.013

(32) Fu, J. R.; Das, D.; Xing, G. L.; Valtchev, V.; Qiu, S. L. J. Am. Chem. Soc. 2016, 138, 7673. doi: 10.1021/jacs.6b03348

(33) McCarthy, M. C.; Varela-Guerrero, V.; Barnett, G. V.; Jeong, H. K.
Langmuir 2010, 26, 14636. doi: 10.1021/1a102409e

(34) Pan, Y. C.; Lai Z. P. Chem. Commun. 2011, 47, 10275. doi: $10.1039 / \mathrm{C} 1 \mathrm{CC} 14051 \mathrm{E}$

(35) Nagaraju, D.; Bhagat, D. G.; Banerjee, R.; Kharul, U. K. J. Mater. Chem. A 2013, 1, 8828. doi: 10.1039/c3ta10438a

(36) Guerrero, V. V.; Yoo, Y.; McCarthy, M. C.; Jeong, H. K. J. Mater. Chem. 2010, 20, 3938. doi: 10.1039/B924536G

(37) Budd, P. M.; Msayib, K. J.; Tattershall, C. E.; Ghanem, B. S.; Reynolds, K. J.; McKeown, N. B.; Fritsch, D. J. Membr. Sci. 2005, 251, 263. doi: 10.1016/j.memsci.2005.01.009

(38) Li, P.; Chung, T. S.; Paul, D. R. J. Membr. Sci. 2013, 432, 50. doi: 10.1016/j.memsci.2013.01.009 